\title{
Sunitinib-induced pyoderma gangrenosum-like skin ulcer
}

\author{
Thomas Kuntz MD, Bijan Koushk-Jalali MD, Alexander Kreuter MD
}

Cite as: CMAJ 2020 May 19;192:E552. doi: 10.1503/cmaj.191165

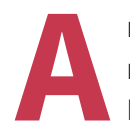

n 80-year-old woman with advanced renal cell carcinoma with multiple pulmonary metastases presented to the Department of Dermatology after a 2-month history of a slowly enlarging, painful ulcer that had occurred after minimal trauma (Figure 1). Several weeks before referral, palliative treatment with sunitinib $(50 \mathrm{mg} / \mathrm{d})$ had been started. The patient had no history of diabetes, venous insufficiency or peripheral arterial disease.

Histopathologic examination of a lesional biopsy found a dense inflammatory infiltrate with neutrophils and erythrocyte extravasation. Bacterial cultures of the lesion showed colonization with Staphylococcus aureus and Proteus mirabilis. We started conventional ulcer therapy with topical antiseptic measures, foam dressings and intermittent curettage of fibrinous coatings, with no significant clinical improvement. Based on the absence of classic causes of leg ulcerations, poor response to conventional therapy and timely correlation of onset of skin ulceration and initiation of sunitinib, we suspected a causal mechanism and therefore stopped sunitinib treatment. Within 4 weeks, the ulcer had completely cleared. However, reintroduction of sunitinib 6 months later led to recurrence of the ulcer at the same site.

Sunitinib is an oral multikinase inhibitor with antiproliferative and antiangiogenic properties. It is approved for renal cell carcinoma, gastrointestinal stromal tumours and pancreatic neuroendocrine tumours. ${ }^{1}$ The most frequent adverse events associated with use of sunitinib are skin lesions, fatigue, diarrhea, nausea and hypertension. Up to $80 \%$ of patients taking multikinase inhibitors such as sunitinib have cutaneous adverse effects, with skin reactions on the hands and feet and stomatitis as the 2 main complications. ${ }^{2}$ Development of pyoderma gangrenosum-like leg ulcers is a rarely reported cutaneous adverse effect of sunitinib. Similar phenomena are described with the use of other biological agents. ${ }^{2,3}$ The pathomechanism of these ulcers is unclear, but antiangiogenic effects by c-kit and VEGFR inhibition that induce skin necrosis and subsequent invasion of neutrophils have been postulated. ${ }^{1}$

Physicians caring for patients with cancer who have skin ulcers and are taking sunitinib should consider this uncommon but potentially severe adverse effect.

\section{References}

1. Lopez Pineiro M, Willis E, Yao C, et al. Pyoderma gangrenosum-like ulceration of the lower extremity secondary to sunitinib therapy: a case report. SAGE Open Med Case Rep 2018;6: $2050313 \times 18783048$.

2. Lee WJ, Lee JL, Chang SE, et al. Cutaneous adverse effects in patients treated with the multitargeted kinase inhibitors sorafenib and sunitinib. Br J Dermatol 2009;161:1045-51.

3. Puig L. Paradoxical reactions: anti-tumor necrosis factor alpha agents, ustekinumab, secukinumab, ixekizumab, and others. Curr Probl Dermatol 2018;53:49-63.

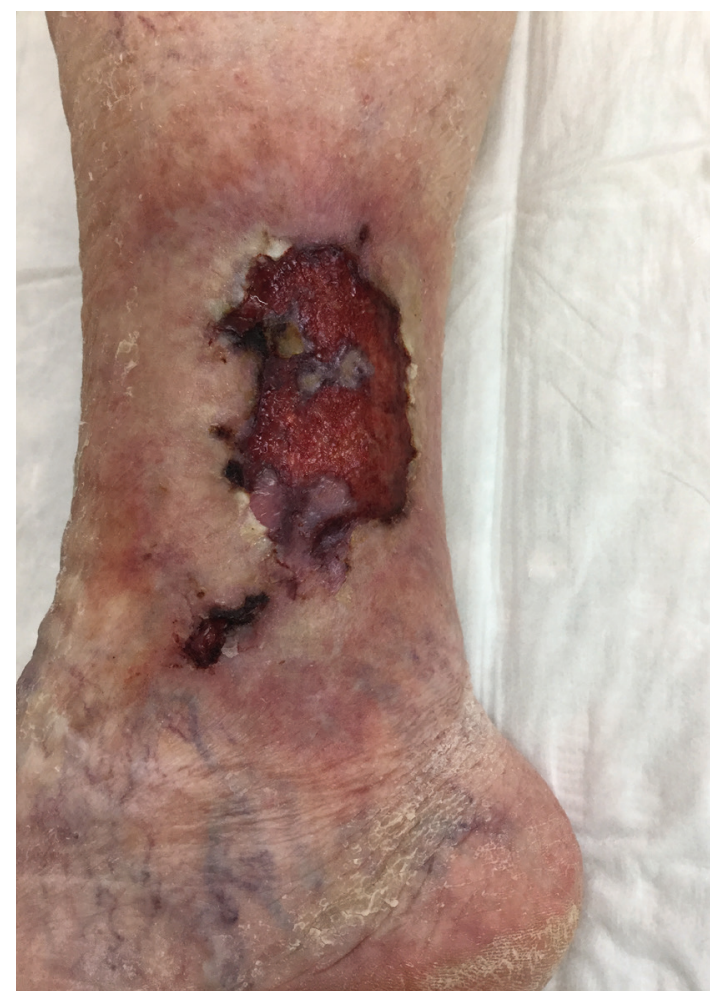

Figure 1: A superficial ulcer (50-80 $\mathrm{mm}$ in diameter) on the right medial malleolus of an 80-year-old woman with renal cell carcinoma who was taking sunitinib. Cutaneous signs of chronic venous insufficiency (corona phlebectatica and mild ankle edema) were present; however, no abnormal blood flow was found on examination using colour ultrasonography.

Competing interests: None declared.

This article has been peer reviewed.

The authors have obtained patient consent.

Affiliation: Department of Dermatology, Venereology, and Allergology, HELIOS St. Elisabeth, Hospital Oberhausen, University Witten-Herdecke, Oberhausen, Nordrhein-Westfalen, Germany

Correspondence to: Alexander Kreuter, a.kreuter@ derma.de 